\title{
Using Statistical Shape Analysis for the Determination of Uterine Deformation States During Hydrometra
}

\author{
M. Harders and G. Székely \\ Virtual Reality in Medicine Group \\ Computer Vision Lab, ETH Zurich \\ CH-8092 Zürich, Switzerland \\ \{mharders, szekely\}@vision.ee.ethz.ch
}

\begin{abstract}
A fundamental prerequisite of hysteroscopy is the proper distension of the uterine cavity with a fluid, also known as hydrometra. For a virtual reality based simulation of hysteroscopy, the uterus deformation process due to different pressure settings has to be modeled. In previous work we have introduced a hybrid method, which relies on precomputed deformation states to derive the hydrometra changes during runtime. However, new offline computations were necessary for every newly introduced organ mesh. This is not viable if a new surgical scene is to be generated for every training session. Therefore, we include the deformation states during hydrometra into our previously developed statistical shape model for undeformed organ instances. This allows deriving the hydrometra steps together with new undeformed uterus meshes. These can then be used during the interactive simulation for predicting uterus deformation without time-intensive precomputation steps.
\end{abstract}

\section{Introduction}

During hysteroscopy [1 - the endoscopic inspection of the uterus - a hydrometra is maintained, i.e. the uterus is distended with liquid media to access the uterine cavity. In- and outflow of the distension fluid is accomplished through the rigid endoscope and controlled via valves, while the pressure of the liquid is provided by a pump. An essential element of the procedure is the selection of correct pressure settings for the hydrometra according to muscle tone and uterine wall thickness [2. In addition to uterus distension, the fluid circulation also improves visibility during the intervention by reducing obscurations caused by endometrial bleeding, small floating tissue fragments or air bubbles. According to [3, a number of complications can be encountered related to the distension fluid handling, for instance fluid overload due to absorption and intravasation, which can have serious adverse effects, possibly leading to cerebral edema or even death. Correct handling of pressure and flow induced by the liquid is therefore a key skill required from gynecological surgeons, thus necessitating appropriate training approaches. 

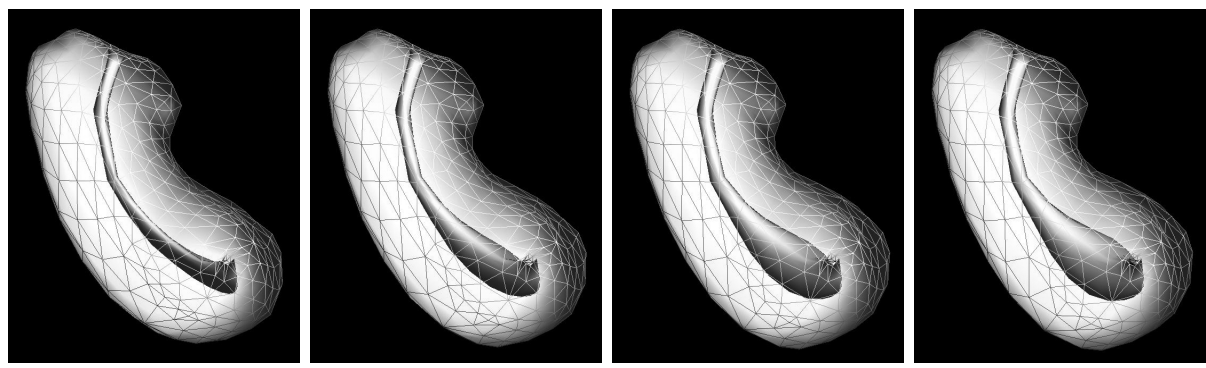

Fig. 1. Side view of uterus triangle mesh (with transparent outer surface) showing computed hydrometra states at increasing pressure of the distension fluid

Virtual reality based surgical simulation [4] offers a promising complementary teaching tool to today's educational paradigms. Our current research targets the development of a high fidelity simulator for procedural training of hysteroscopy [5], addressing key elements such as proper cavity inflation and correct management of fluid pressure settings. A core module of the simulation system is the real-time uterine deformation during the varying hydrometra stages. The almost instantaneous response of the uterine cavity to pressure adjustments of the distension fluid exceeds by far the real-time capabilities of most known deformation models. Therefore, we have suggested in [6] a novel approach, which combines accurate Finite Element Method (FEM) computations with Free-Form Deformation (FFD) approaches to achieve physically realistic real-time distension of the uterine cavity. In the proposed method, first offline precomputations are carried out to determine an accurate response of the tissue model to the fluid pressure. Thereafter, these data are used during real-time interaction for model adjustments based on given pressure states.

Unfortunately, merging this method with our training scene generation framework [7] posed some problems. The underlying idea of the latter is to prevent the trainee from repeatedly being confronted with the same surgical scene and thus to avoid becoming acquainted with a specific anatomy. Therefore, the generation of a new scenario for each single training session considers the full natural variability of the healthy anatomy. Based on a predefined set of anatomical gynecological measurements, new surface meshes of the uterus can be intuitively derived prior to the training. However, following the previously mentioned strategy, every new geometry would require the offline precomputation of the varying hydrometra states via FEM. This usually requires significant manual processing to setup the new loadcases. Moreover, depending on the mesh resolution, considerable computation times can result. For instance in [8] it is reported that 40 load steps for the inflation of a 56'000 element uterus mesh required about 17 hours of CPU time on a Superdome HP 9000 Enterprise application server (2.2 GFLOPS peak). Finally, it should also be mentioned that appropriate FE software needs to be available to perform the actual computations. In order to avoid all these problems, we propose to extend our statistical shape model of the uterus to also include uterine deformation states during hydrometra. By 

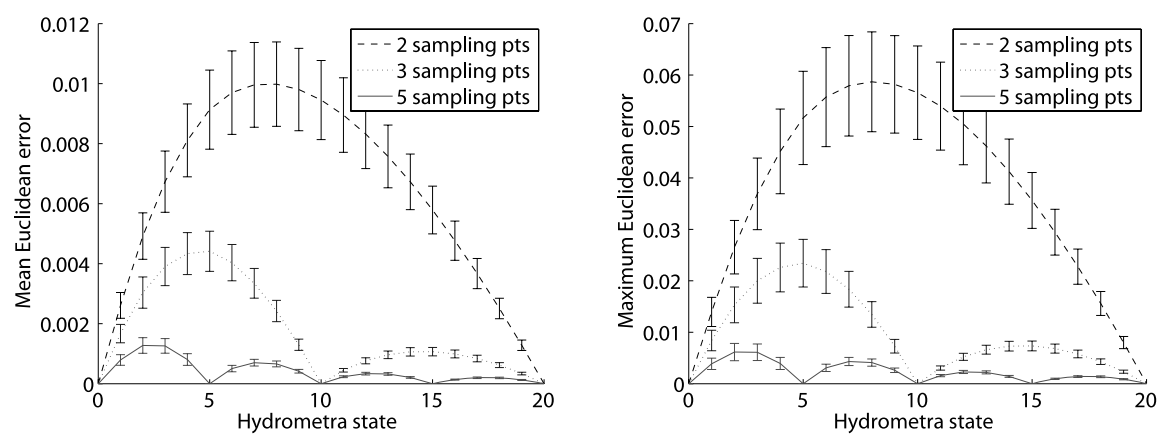

Fig. 2. Influence of number of interpolation states on mean (left) and maximum (right) Euclidean error $[\mathrm{cm}]$. Mean and standard deviation for all organ instances at all deformation states are depicted.

extending the parameter vector with the deformed shapes, new instances of the hydrometra steps can be generated directly via the statistical shape model. Related activities have been carried out using statistical models in cardiac motion analysis (e.g. 9]) or longitudinal human bone growth studies (e.g. for mandibles [10]). In [11 a similar technique has been applied to estimate the shape of a prostate phantom deformed during transrectal ultrasound probe insertion. This work focuses, however, on deriving a patient-specific model for intra-operative support and the input data are based only on a single simplified anatomical phantom.

\section{Method}

\subsection{Computation of Deformation States}

The first step in our approach is the computation of the deformation states. As starting point we use $N=16$ triangle meshes of healthy organ geometries (given by polygonal models $\hat{\mathbf{p}}_{i}=\left[x_{i}^{[1]}, y_{i}^{[1]}, z_{i}^{[1]}, \ldots, x_{i}^{[M]}, y_{i}^{[M]}, z_{i}^{[M]}\right]^{T}$, with $M=630$ vertices) which were segmented from MRI data obtained in a volunteer study. During the segmentation step correspondences between mesh vertices are implicitly ensured by starting with the same coarse organ mesh for all uteri initialized according to surface landmarks and following a fixed subdivision scheme during segmentation [12, resulting in identical mesh topologies $\Theta$. All shapes were translated and rotated into a common organ coordinate system, however, no normalization was applied in order to also capture organ size differences. Consistent tetrahedral meshes for these surface representations are then automatically generated using the commercial ANSYS ICEM CFD Tetra Mesher tool. Thereafter, FEM computation of the hydrometra states are carried out based on the resulting tetrahedral models. Note that our presented statistical model would work with arbitrary deformation algorithms and that the presented FEM computation is only one possible approach to obtain the hydrometra states. The uterine 

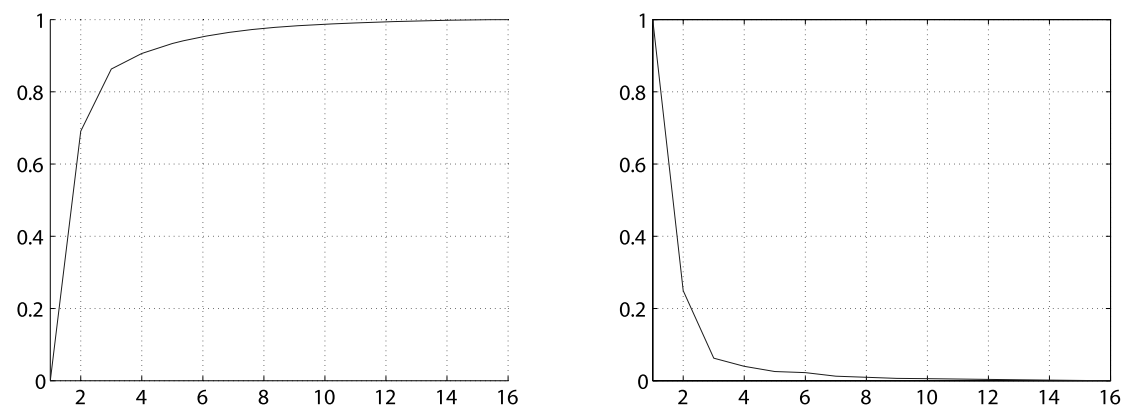

Fig. 3. Shape variability captured by the first eigenmodes, plotted by $k \mapsto$ $\left(\sum_{l=1}^{k} \lambda_{l} / \sum_{l=1}^{N-1} \lambda_{l}\right.$ (left) and normalized variances of decorrelated shape parameters, plotted by $k \mapsto \frac{\lambda_{k}}{\lambda_{1}}($ right)

soft tissue is represented as a homogeneous, isotropic and nonlinear hyper-elastic material, with the polynomial strain energy function given by

$$
W=\mu_{1}\left(J_{1}-3\right)+\mu_{2}\left(J_{1}-3\right)^{3}+\frac{1}{2} \kappa\left(J_{3}-3\right)^{2}
$$

where the $J_{i}$ are the reduced invariants of the right Cauchy-Green deformation tensor, the $\mu_{i}\left[\mathrm{~N} / \mathrm{m}^{2}\right]$ are the material parameters and $\kappa\left[\mathrm{N} / \mathrm{m}^{2}\right]$ is the bulk modulus [13. The material parameters are determined according to the in-vivo tissue aspiration experiment described in [13, while the bulk modulus is set to $\kappa=10^{7} \mathrm{~N} / \mathrm{m}^{2}$ in order to model quasi-incompressible behavior. The validity of this material description has been experimentally shown in 8] by comparison with in-vivo measurements. The vertices around the outer cervical and tubal ostia are fixed to provide appropriate boundary conditions, while the remaining nodes are free to move. Hydrostatic pressure face loads are applied to all surfaces of the uterine cavity. Note that due to the correspondences between the meshes, coherent boundary conditions can be applied to all segmented models. 20 organ deformation states $\left\{\mathbf{q}_{i}^{j} \mid j \in\{1, \ldots, 20\}\right\}$ are obtained for increasing hydrostatic pressures of discrete steps of $1 \mathrm{kPa}$ up to $20 \mathrm{kPa}$ (about $150 \mathrm{mmHg}$ ) for all organ models $\hat{\mathbf{p}}_{i}$. These FEM computations are carried out with the commercial package MarcMentat ${ }^{T M}$. A subset of the resulting hydrometra stages are depicted for one exemplary uterus model in Figure 1.

\subsection{Reduction of Parameter Space}

As described in [6], the final goal of our endeavor is to import the precomputed deformation states into our hysteroscopy simulator, in order to determine at runtime the shape of the uterine cavity by interpolating between them according to the interactively modified fluid pressure. Qualitative findings of our earlier work indicated that the overall deformation process could be approximated sufficiently well based on a small subset of the computed hydrometra states. In 

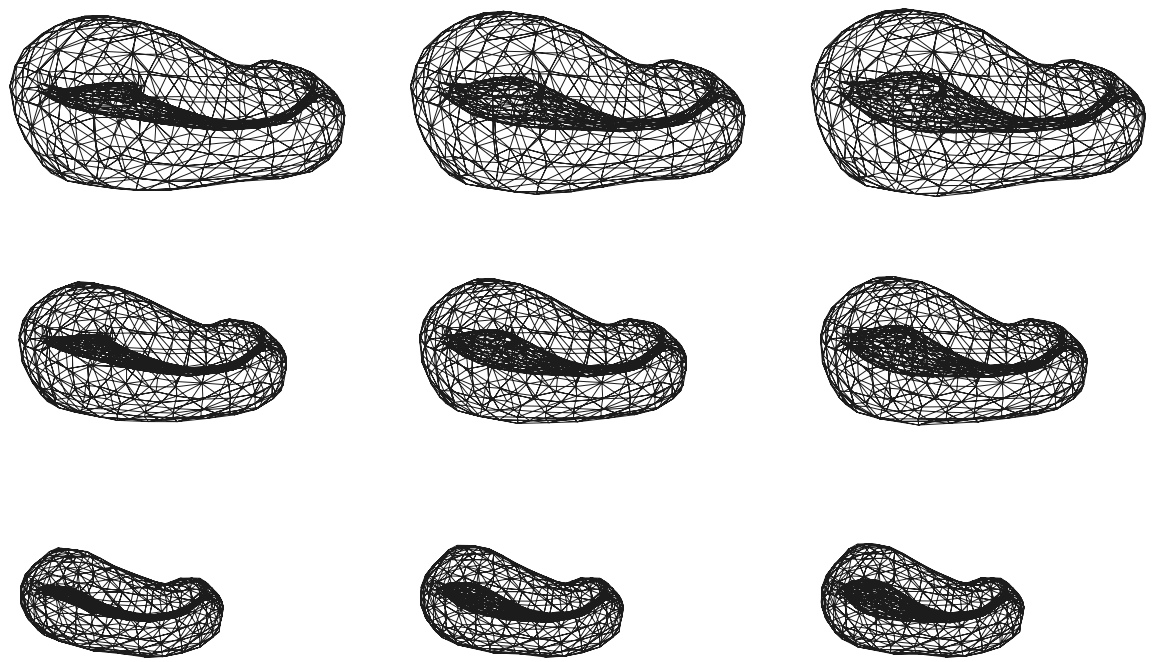

Fig. 4. The first eigenmode of the extended statistical uterus model captures the overall size of the uterus. Both the undeformed (left) and the deformed states (middle: $j_{1}=10$, right: $j_{2}=20$ ) are obtained by evaluating $\overline{\mathbf{p}}+\omega \sqrt{\lambda_{1}} \mathbf{u}_{1}$ with $\omega \in\{-2,0,2\}$ (top to bottom).

order to quantify the influence of the number of deformation states included in the piecewise linear interpolation, we examined the Euclidean error between the vertices of interpolated hydrometra states and the computed deformations. Figure 2 shows mean and standard deviation of the average and maximum errors of all organ instances at all deformation states, where state 0 denotes the undeformed organ mesh. When compared to the mean of the average edge lengths of all meshes of $0.403 \mathrm{~cm}(\sigma=0.0503 \mathrm{~cm})$ and the mean of all minimal edge lengths of $0.0331 \mathrm{~cm}(\sigma=0.0054 \mathrm{~cm})$, using three states already appears to be sufficiently precise. Therefore, in the following only a reduced set of hydrometra states will be taken into account. Nevertheless, the described method is of course also valid for a larger number of deformation states. Finally, it should be noted that slightly larger errors occur in the early stages of the uterus' extension. Therefore, the interpolation errors could potentially be further decreased by an appropriate selection of less uniformly distributed samples. However, due to the already small error this issue was not further investigated.

\subsection{Statistical Model of Hydrometra States}

In the next step we combine the vertices of an undeformed uterus shape and the reduced set of hydrometra states into one instance specific shape vector $\mathbf{p}_{i}=$ $\left[\hat{\mathbf{p}}_{i} \mathbf{q}_{i}^{j_{1}} \ldots \mathbf{q}_{i}^{j_{s}}\right]^{T}$ where $\mathcal{S}=\left\{j_{1}, j_{2}, \ldots, j_{s}\right\}$ denotes the reduced set of indices of selected deformation states. By computing the instance specific differences from the respective mean shapes we obtain the $s M \times N$ matrix $\Delta \mathbf{P}=\left[\Delta \mathbf{p}_{1} \ldots \Delta \mathbf{p}_{N}\right]$ with $\Delta \mathbf{p}_{i}=\mathbf{p}_{i}-\overline{\mathbf{p}}$. The matrix $\Delta \mathbf{P}$ is rank deficient, since $s M \gg N$; only a set 

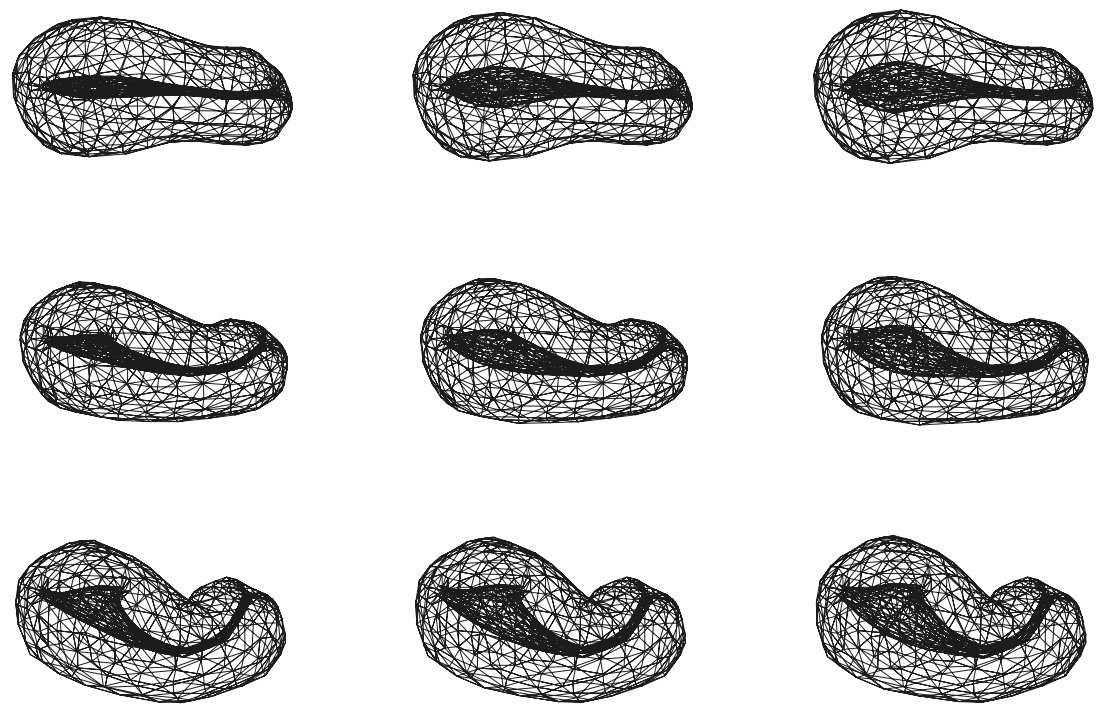

Fig. 5. The second eigenmode of the extended statistical uterus model represents the change of angle between cervix and fundus. Both the undeformed (left) and the deformed states (middle: $j_{1}=10$, right: $j_{2}=20$ ) are obtained by evaluating $\overline{\mathbf{p}}+\omega \sqrt{\lambda_{2}} \mathbf{u}_{2}$ with $\omega \in\{-2,0,2\}$ (top to bottom).

of $N-1$ eigenvalues $\boldsymbol{\Lambda}$ of the corresponding covariance matrix will be non-zero. Therefore, we follow the alternative approach discussed in [14 to determine the corresponding set of eigenvectors $\mathbf{U}$, by performing the singular value decomposition of the reduced $N \times N$ covariance matrix

$$
\tilde{\mathbf{\Sigma}}=\frac{\mathbf{1}}{\mathbf{N}-\mathbf{1}} \Delta \mathbf{P}^{\mathbf{T}} \boldsymbol{\Delta} \mathbf{P} \stackrel{P C A}{=} \tilde{\mathbf{U}} \tilde{\Lambda} \tilde{\mathbf{U}}^{T} .
$$

The sought-after eigenvectors can then be determined according to $\mathbf{U}=$ $((N-1) \lambda)^{-\frac{1}{2}} \boldsymbol{\Delta} \mathbf{P} \tilde{\mathbf{U}}$. The scaling factor is necessary to normalize the vectors. The corresponding eigenvalues are given directly by $\boldsymbol{\Lambda}=\tilde{\boldsymbol{\Lambda}}$. As depicted in Figure 3. the first four eigenmodes already capture $94 \%$ of the overall variation in the test collection. Thus, our set of undeformed and deformed organ triangle meshes can be compactly described by a reduced set of eigenvectors $\breve{\mathbf{U}} \subset \mathbf{U}$. By evaluating $\overline{\mathbf{p}}+\breve{\mathbf{U} b}$ vertices of new organ instances and their associated hydrometra states can be derived. Due to space limitations, only a subset of the variation of the two most dominant eigenmodes of the considered population are displayed in Figures 4 and 5.

\section{Discussion}

In order to assess the performance of our approach, we derived new instances of organ shapes $(\bar{N}=11)$ and the associated hydrometra states by determining 

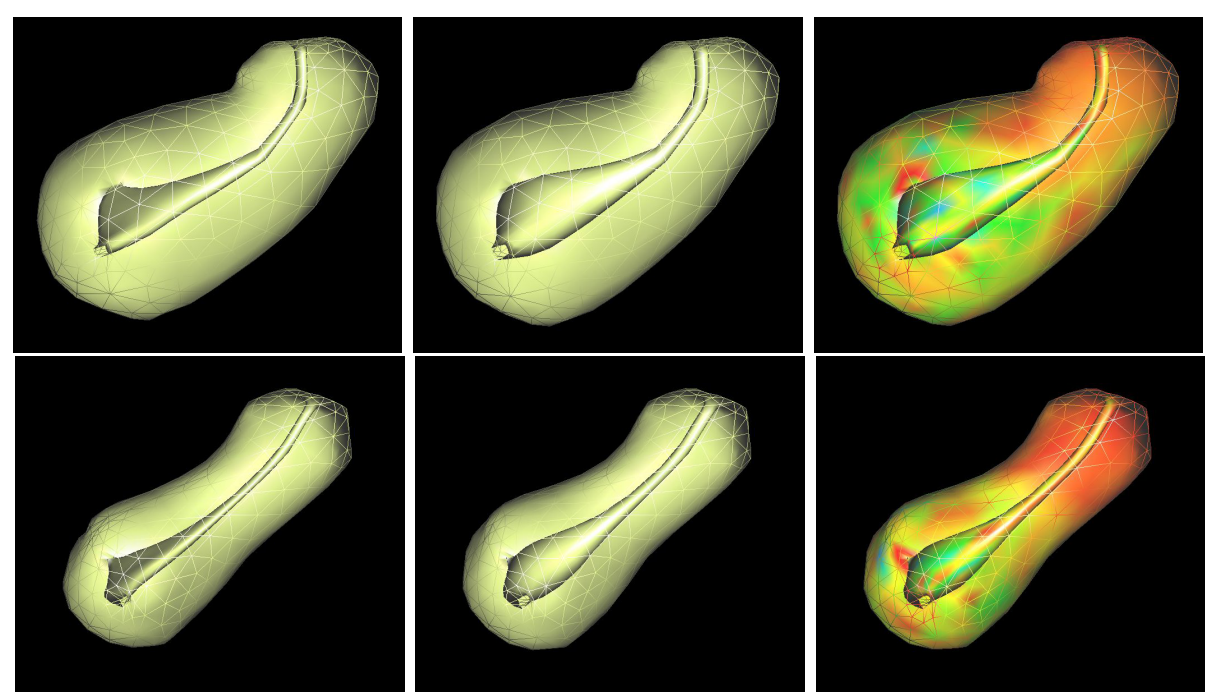

Fig. 6. Comparison of automatically derived (middle) and computed (right) hydrometra deformation states for two undeformed uterine meshes (left). The Euclidean error between the meshes is shown color-coded $(r e d=0 \mathrm{~cm})$. A maximal error of $0.03996 \mathrm{~cm}$ and $0.04827 \mathrm{~cm}$ results.

$\overline{\mathbf{p}}+\sum_{k=1}^{4} \omega_{k} \sqrt{\lambda_{k}} \mathbf{u}_{k}$ with weighting parameter $-2 \leq \omega_{k} \leq 2$. The latter are then compared to FEM deformation calculations based on the undeformed meshes. A mean maximal vertex distance error of $0.0353 \mathrm{~cm}(\sigma=0.0081 \mathrm{~cm})$ results, which is on the order of the minimal edge lengths in the meshes. Figure 6 shows two examples of uterine shapes for which the maximum hydrometra distension is derived with our method and compared to a computed deformation state. The maximum error is $0.03996 \mathrm{~cm}$ and $0.04827 \mathrm{~cm}$, respectively.

It should be mentioned that the proposed approach based on precomputation of deformation states is only valid, if the boundary conditions do not considerably change during the intervention. This is usually the case in hysteroscopic procedures, since the surrounding tissues are not directly accessible. Moreover, extreme modifications of the cavity are not possible in our system, since cutting into the myometrium is limited to a thin layer during endometrial ablation. Therefore, we can assume, that the uterus' response remains relatively constant throughout the intervention. The piecewise linear interpolation allows to update large meshes consisting of more than 50'000 tetrahedra in real-time.

\section{Conclusion and Future Work}

We have presented an extension to our statistical shape model of the uterus to include uterine deformation states during hydrometra. By extending the training set with the deformed shapes, new instances of the hydrometra states can be generated directly by the statistical shape model. This avoids explicit time-intensive 
precomputations for newly derived organ instances. Our validation study demonstrated that the deformations predicted by the extended statistical model are in good agreement with the results of nonlinear FEM calculations. The behavior of the uterus under changing fluid pressure settings can be well approximated by linear interpolation in real-time, even for large meshes.

\section{Acknowledgment}

This work has been performed within the frame of the Swiss National Center of Competence in Research on Computer Aided and Image Guided Medical Interventions (NCCR CO-ME) supported by the Swiss National Science Foundation.

\section{References}

1. Bulletin, A.T.: Hysteroscopy. Int. J. Gyn. Obstet. 45(2), 175-180 (1994)

2. Petrozza, J.: Hysteroscopy (2004), http://www.emedicine.com/med/topic3314.html

3. Mencaglia, L., Hamou, E.: Manual of gynecological hysteroscopy - diagnosis and surgery. Endo-Press, Germany (2001)

4. Basdogan, C., Sedef, M., Harders, M., Wesarg, S.: Virtual reality supported simulators for training in minimally invasive surgery. IEEE Computer Graphics and Applications 27, 54-66 (2007)

5. Harders, M., Bajka, M., Spaelter, U., Tuchschmid, S., Szekely, G.: Highly-realistic, immersive training environment for hysteroscopy. In: Proc. of Medicine Meets Virtual Reality, pp. 176-181 (2006)

6. Sierra, R., Zátonyi, J., Bajka, M., Székely, G., Harders, M.: Hydrometra simulation for vr-based hysteroscopy training. In: Duncan, J.S., Gerig, G. (eds.) MICCAI 2005. LNCS, vol. 3749, pp. 575-582. Springer, Heidelberg (2005)

7. Sierra, R., Bajka, M., Karadogan, C., Székely, G., Harders, M.: Coherent scene generation for surgical simulators. In: Cotin, S., Metaxas, D.N. (eds.) ISMS 2004. LNCS, vol. 3078, pp. 221-229. Springer, Heidelberg (2004)

8. Weiss, S., Bajka, M., Nava, A., Mazza, E., Niederer, P.: A finite element model for the simulation of hydrometra. Technology and Health Care 12(3), 259-267 (2004)

9. Chandrashekara, R., Rao, A., Sanchez-Ortiz, G., Mohiaddin, R., Rueckert, D.: Construction of a statistical model for cardiac motion analysis using nonrigid image registration. Inf. Process Med. Imaging, 599-610 (2003)

10. Andresen, P., Bookstein, F., Conradsen, K., Kreiborg, S.: Surface-bounded growth modeling human mandibles. IEEE Transactions on Medical Imaging 19 (2000)

11. Mohamed, A., Davatzikos, C., Taylor, R.: A combined statistical and biomechanical model for estimation of intra-operative prostate deformation. In: Dohi, T., Kikinis, R. (eds.) MICCAI 2002. LNCS, vol. 2488, pp. 452-460. Springer, Heidelberg (2002)

12. Sierra, R., Zsemlye, G., Szekely, G., Bajka, M.: Generation of variable anatomical models for surgical training simulators. Medical Image Analysis 10(2), 275-285 (2006)

13. Kauer, M., Vuskovic, V., Dual, J., Szekely, G., Bajka, M.: Inverse finite element characterization of soft tissues. Medical Image Analysis 6(3), 275-287 (2002)

14. Cootes, T., Cooper, D., Taylor, C., Graham, J.: Active shape models - their training and application. Computer Vision and Image Understanding 61(1), 38-59 (1995) 